\title{
Non-woven shape-memory polymer blend actuators
}

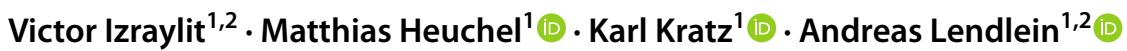

Received: 26 March 2021 / Accepted: 28 April 2021 / Published online: 25 June 2021

(c) Helmholtz-Zentrum Hereon 2021

\begin{abstract}
The hierarchical design approach provides various opportunities to adjust the structural performance of polymer materials. Electrospinning processing techniques give access to molecular orientation as a design parameter, which we consider here in view of the shape-memory actuation performance. The aim of this work is to investigate how the reversible strain $\varepsilon_{\text {rev }}^{\prime}$ can be affected by a morphology change from a bulk material to an electrospun mesh. $\varepsilon_{\text {rev }}^{\prime}$ could be increased from $5.5 \pm 0.5 \%$ to $15 \pm 1.8 \%$ for a blend from a multiblock copolymer with poly $(\varepsilon$-caprolactone) (PCL) and poly ( $L$-lactide) (PLLA) segments with oligo( $D$-lactide) (ODLA). This study demonstrates an effective design approach for enhancing soft actuator performance, which can be broadly applied in soft robotics and medicine.
\end{abstract}

\section{Introduction}

Soft polymeric actuators are capable to perform a reversible movement controlled by external stimuli. Tunability and reprogrammability distinguish shape-memory polymer actuators from other types of soft actuators [1]. The physical principle underlying the shape-memory actuation capability is the tendency of polymer molecules in actuator domains to form crystallites along their orientation, which is maintained by a skeleton of a second set of crystallites associated to a higher melting temperature than the actuating crystallites, and their entropy driven contraction upon melting [2]. In the ideal case, the highest shape-memory performance $\varepsilon_{\text {rev }}^{\prime}$ is achieved at the maximal molecular orientation. In a shape-memory polymer actuator, the molecular orientation is typically achieved by deforming the material during the programming procedure. Its degree is however limited due to the stochastic spatial distribution of cross-links and entanglements spacing in bulk samples [3]. Attempts to achieve higher orientation can lead to disintegrating of the netpoints and disentangling of the network $[4,5]$.

\section{Deceased: Karl Kratz.}

Andreas Lendlein

lendlein@uni-potsdam.de

1 Institute of Active Polymers, Helmholtz-Zentrum Hereon, Kantstr. 55, 14513 Teltow, Germany

2 Institute of Chemistry, University of Potsdam, Karl-Liebknecht-Str. 24/25, 14476 Potsdam, Germany
A viable approach to improve molecular orientation is to implement a processing technique, which allows for simultaneous aligning of the polymer molecules and cross-linking. A challenge here is to avoid slippage of chains against each other by relying on entanglements as the netpoints, introducing temporary cross-links or conducting the cross-linking process in situ. For instance, drawing a polymeric fiber out of an extruder die and its simultaneous cooling can develop molecular orientation, which is further fixed by crystallisation and finally consolidated by cross-linking with gamma ray irradiation [6]. Such fibers demonstrate a shape-memory actuation capability and require no additional programming. An alternative processing technique to achieve higher degrees of molecular orientation is electrospinning. Here, high-voltage applied to a polymer solution generates inside it an electrostatic repulsion, which overcomes the surface tension and forms a jet at the solution surface. The erupting solution accelerates in the electrostatic field and dries on its flight to the collector, forming fibers of diameters down to hundreds of $\mathrm{nm}$. This approach allows for producing highly oriented microfibers, where the degree of orientation increases with the reduction of fiber diameter [7]. Similarly to the extruded fibers, the molecular orientation in the electrospun meshes can be maintained by temporary intermolecular interactions and permanently fixed by subjecting to UV-irradiation or acid vapours [8,9]. The resulting crosslinked fiber meshes demonstrate the shape-memory actuation capability under the stress-free conditions. Due to the fragility of micro- and nanofibers, cross-linking of electrospun fiber meshes with common post-processing techniques 
can result in their sintering, losing of the preorientation, and so compromising their mechanical integrity $[10,11]$.

The necessity for post-processing treatment can be omitted by introducing an in situ cross-linking technique. The trigger for initiating the cross-linking in the course of electrospinning is evaporation of solvent. Such interaction can be provided by polylactide (PLA) stereocomplexation, an interaction between opposite enantiomers, organising them into a periodic crystalline structure [12]. We have previously demonstrated that blending of a multi-block copolymer containing poly(L-lactide) and poly( $\varepsilon$-caprolactone) segments (PLLA-PCL) with oligo(D-lactide) (ODLA) in a one-step solution-casting process leads to formation of a physical network [13]. PLLA-PCL/ODLA blends have been validated to be capable of the shape-memory actuation capability. In this study, we investigate the effect of sample morphology on $\varepsilon_{\text {rev }}^{\prime}$ of the shape-memory actuation capability. Thermal properties and crystalline structure were analysed with DSC and WAXS. The morphology of the electrospun meshes was examined with SEM. The shape-memory actuation capability was quantified in cyclic thermomechanical experiments.

\section{Materials and methods}

\section{Materials and processing}

A multi-block copolymer containing poly(L-lactide) and poly ( $\varepsilon$-caprolactone) segments (PLLA-PCL) was synthesized as reported recently [13]. PLLA-PCL has a segment structure out of 12 repeating units of L-lactide and 58 repeating units of $\varepsilon$-caprolactone. The molecular weight is $M_{\mathrm{n}}=200 \mathrm{~kg} \cdot \mathrm{mol}^{-1}$. Oligo $(D$-lactide) (ODLA) was reported to have $M_{\mathrm{n}}=1.8 \mathrm{~kg} \cdot \mathrm{mol}^{-1}$.

Films were prepared via solution casting. A PLLA-PCL/ ODLA mixture in $90 / 10 \mathrm{wt} \%$ ratio was dissolved at a predetermined ratio in chloroform $(99.9 \%$, Carl Roth, Karlsruhe, Germany). The solution was stirred for $3 \mathrm{~h}$ until the polymers were dissolved, then left to evaporate in a covered polytetrafluoroethylene (PTFE) petri dish in ambient conditions for 2 days.

Unwoven meshes of a PLLA-PCL/ODLA mixture in 90/10 wt\% ratio were prepared using an electrospinning setup (Linari Engineering, Pisa, Italy) equipped with a voltage supply, rotatory drum collector (diameter: $8 \mathrm{~cm}$ ) and syringe pump. The process was performed inside a ventilated chamber with humidity kept in a range of $h=15-20 \%$. The solutions were prepared in tetrahydrofuran (THF, 99.8\%, Carl Roth, Karlsruhe, Germany) in a concentration range of $C=1-10 \%$ w/v. The solution was ejected through a needle with inner diameter of $0.8 \mathrm{~mm}$. The potential difference, applied between the nozzle and the collector drum, was manually controlled as $\Delta U=10-13 \mathrm{kV}$ to achieve a stable flow. The solution flow rate was maintained at $f=2.1 \mathrm{~mL} \cdot \mathrm{h}^{-1}$ throughout the course of electrospinning. The rotation of the collector was set to $10 \mathrm{rpm}$ and the distance between the nozzle and the collector was $25 \mathrm{~cm}$. The yarn samples were manufactured by manual winding of the electrospun meshes.

\section{Scanning electron microscopy (SEM)}

Scanning electron microscopy (SEM) was applied to analyse the fiber diameter and arrangement. The experiments were performed on a Phenom G2 Pro (Thermo Fisher Scientific, Waltham, USA). The single fiber diameter was calculated as average of 20 measurements. The fibers were coated with a conductive gold layer on a sputter coater Polaron SC7640 (Hertfordshire, England). A voltage of $1-3 \mathrm{kV}$ was applied during characterization. Alignment of the fibers was characterised with ImageJ (National Institute of Health, Bethesda, USA).

\section{Thermal characterisation}

Differential scanning calorimetry (DSC) experiments were conducted on a Netzsch DSC 204 Phoenix (Selb, Germany) at heating and cooling rates of $10{ }^{\circ} \mathrm{C} \cdot \mathrm{min}^{-1}$ in pierced aluminium pans. For determining the thermal properties of the polymers and blends, the materials were scanned in the temperature range -100 to $200{ }^{\circ} \mathrm{C}$. The crystallinity of all components was calculated according to the equation:

$\chi_{\mathrm{c}}=\frac{\Delta H_{\mathrm{m}}}{\Delta H_{\mathrm{m}}^{100}} \times \frac{1}{W} \times 100$,

where $\Delta H_{\mathrm{m}}$ is the experimental melting enthalpy of a fraction, determined as the area under the melting peak. $\Delta H_{\mathrm{m}}^{100}$ is the specific melting enthalpy of $100 \%$ crystalline polymer, which is $135 \mathrm{~J} \cdot \mathrm{g}^{-1}$ for PCL [14] and $142 \mathrm{~J} \cdot \mathrm{g}^{-1}$ for PLA stereocomplex [12]. $W$ is the weight content of the fraction in the copolymer of the blend. The PLA stereocomplex fraction was calculated as the maximal amount of coupling $\mathrm{L}$ - and D-lactide units. The data were registered at the first heating and cooling runs.

\section{X-ray diffraction analysis}

Wide angle X-ray scattering (WAXS) measurements were performed with a D8 Discover spectrometer with a 2D-detector (Bruker AXS, Karlsruhe, Germany) at the ambient temperature. The samples with an area of $2 \times 0.5 \mathrm{~cm}^{2}$ and a thickness of about $150 \mu \mathrm{m}$ were fixed at both ends during characterization. 


\section{Thermomechanical testing}

All mechanical tests were performed using a thermomechanical tensile tester Zwick Z1.0 (Ulm, Germany) equipped with a thermo-chamber and a temperature controller. A clamping distance of $10 \mathrm{~mm}$ was used in all of the experiments. Elongation at break $\varepsilon_{\text {break }}$ of the samples was tested by stretching the samples at defined temperatures and a constant deformation rate of $5 \mathrm{~mm} \cdot \mathrm{min}^{-1}$ until breakage occurred.

The actuation capability of the material was characterized by programming, followed by three repetitive actuation cycles. The sample was heated in the thermo-chamber at the programming temperature $T_{\text {prog }}=70^{\circ} \mathrm{C}$ for $10 \mathrm{~min}$ to remove the thermal history in the PCL segments. Then, the sample was elongated to the programming strain $\varepsilon_{\text {prog }}=500 \%$ at $5 \mathrm{~mm} \cdot \mathrm{min}^{-1}$. The sample was held at $T_{\text {prog }}$ and $\varepsilon_{\text {prog }}$ for $5 \mathrm{~min}$ to allow the initial relaxation processes, i.e., the alignment of polymer chains along the deformation axis. The temporary shape was fixed at $0{ }^{\circ} \mathrm{C}$ under constant force. Then, the force applied to the sample was reduced to $0.05 \mathrm{~N}$, at which the zero-force shape-memory actuation experiment was carried out. The actuation cycle was performed in a temperature window of $T_{\text {low }}=0{ }^{\circ} \mathrm{C}$ and $T_{\text {high }}=55^{\circ} \mathrm{C}$, defined as optimal conditions in the previous work [13]. Actuation performance $\varepsilon_{\text {rev }}^{\prime}$ was calculated as:

$\varepsilon_{\mathrm{rev}}^{\prime}=\frac{l_{\mathrm{b}}-l_{\mathrm{a}}}{l_{\mathrm{a}}} \times 100 \%=\frac{\varepsilon_{\mathrm{b}}-\varepsilon_{\mathrm{a}}}{\varepsilon_{\mathrm{a}}+100} \times 100 \%$,

where $l_{\mathrm{a}}$ and $\varepsilon_{\mathrm{a}}$ are the length of the sample and its engineering strain at the beginning of the actuation cycle at $T_{\text {high }}$ and $l_{\mathrm{b}}$ and $\varepsilon_{\mathrm{b}}$ are the length of the sample and its engineering strain at the beginning of the actuation cycle at $T_{\text {low }}$. The $\varepsilon_{\mathrm{rev}}^{\prime}$ values were calculated as an average in three subsequent cycles.

\section{Results and discussion}

\section{Morphology, structure and thermal properties}

The high molar mass of the studied PLLA-PCL $M_{\mathrm{n}}=200 \mathrm{~kg} \cdot \mathrm{mol}^{-1}$ effected high viscosities of its THF solutions used in electrospinning even at $C \leq 10 \% \mathrm{w} / \mathrm{v}$. Further, the evaporation of the solvent led to a rapid formation of PLA stereocomplexes in the electrospun solutions of PLLAPCL/ODLA blends. This caused clogging of the solution inside the nozzle and its uneven flow. Lowering the solution viscosity by reducing its concentration below a certain threshold value induced the instability of the jet and consequently formation of beads, hollow fibers collapsing into ribbons and branching fibers [15]. These defects in the fiber morphology led to mechanical instability of the non-woven meshes and their abrupt failure under stress. Though other solvents were considered, PLLA-PCL/ODLA fibers electrospun from THF solutions possessed the lowest amount of the described defects. Here, $C=7 \%$ w/v. was experimentally determined as optimal for production of cylindrical fibers with an average diameter of $d=1.8 \pm 0.6 \mu \mathrm{m}$. Due to the high $M_{\mathrm{n}}=200 \mathrm{~kg} \cdot \mathrm{mol}^{-1}$ of PLLA-PCL and consecutive high viscosity of its solutions, the electrospinning throughput was low. A mesh with thickness of $h=50 \pm 2 \mu \mathrm{m}$ was produced in $8 \mathrm{~h}$. Further, the amount of accumulated defects increased, compromising the integrity of the meshes. An exemplary morphology of an electrospun mesh before and after programming for shape-memory actuation is shown in Fig. 1a, b. The respective twisted electrospun yarn is shown in Fig. 1c.

Frequently, formation of stereocomplex or improvement of its crystallinity requires additional post-treatment, e.g., annealing, which can be disastrous for fiber meshes. In our previous work, PLA stereocomplexation between PLLAPCL and ODLA was observed in a one-step process of solution casting [13]. Analogously, PLA opposite enantiomers in the PLLA-PCL/ODLA blend underwent stereocomplexation in the electrospinning process. No PLA isotactic crystallisation was observed in films and electrospun meshes, which was observed by WAXS as an absence of the characteristic PLA scattering maximum at $16^{\circ}$ [16]. (Fig. 1d). This is mainly the result of the PLLA-PCL molecular design and indicates good compatibility of PLLA-PCL/ODLA blend components. Interestingly, no direct evidence of molecular preorientation in the fiber meshes were observed. 2D WAXS scattering patterns are isotropic and demonstrate no preferential scattering direction, which is however typical for meshes of randomly aligned fibers [17] (Insert in Fig. 1d). Orientation-induced increase of crystallinity was not observed with DSC. Here, $\chi_{\mathrm{c}}$ of the PCL domains are $40 \pm 4 \%$ and $36 \pm 4 \%$ in a PLLA-PCL/ODLA film and fiber mesh respectively, while $\chi_{\mathrm{c}}$ of the PLA stereocomplex domains are $48 \pm 5 \%$ and $44 \pm 4 \%$. The PLLA-PCL / ODLA fiber meshes rapidly shrink when heated to $100{ }^{\circ} \mathrm{C}$ indicating the presence of confined stresses.

\section{Shape-memory actuation behaviour}

Oriented polymer chains of the actuator domains form organised arrays of crystallites aligned with the direction of their orientation. Upon melting, they attempt to occupy a highly entropic state of a random coil. The difference between geometrical configurations of these states governs the macroscopic shape change. The collective and directed character of the conformational changes in highly oriented polymers maximises this effect. Thermomechanical treatment of the electrospun meshes at the programming step of 


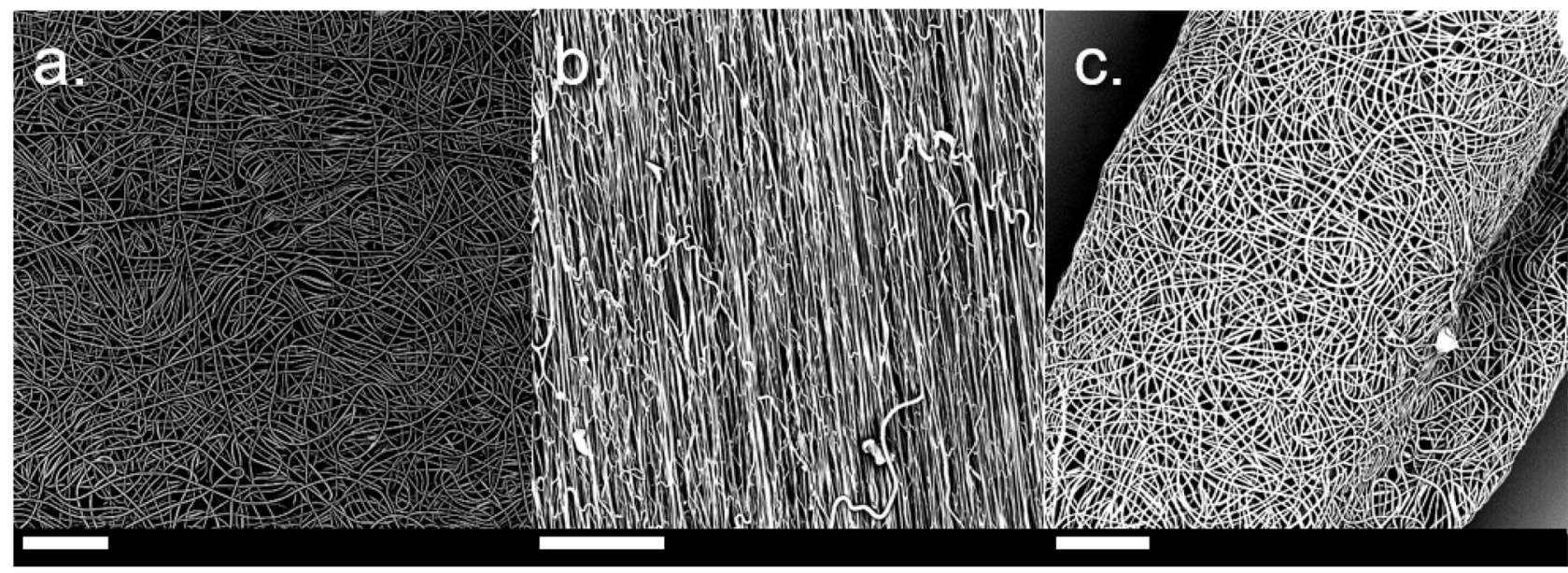

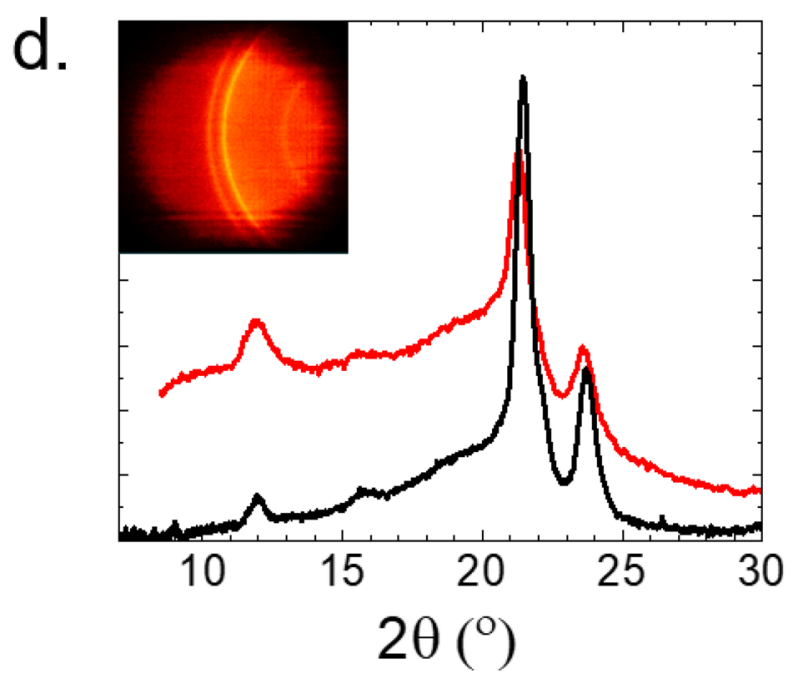

Fig. 1 SEM images of PLLA-PCL/ODLA non-woven materials. a Flat pristine electrospun mesh; b stretched electrospun mesh; c twisted electrospun yarn. Scale bar $100 \mu \mathrm{m}$. d WAXS spectra of PLLA-PCL/ODLA. Black-solution-casted film, red-electrospun mesh, insert-2D scattering image of the electrospun mesh show-

the shape-memory actuation simultaneously aligns the preoriented fibers along the programming direction with a dispersity of directionality distribution of 3.75 and implements a higher degree of orientation upon them. This is observed as an increase in $\varepsilon_{\mathrm{rev}}^{\prime}=5.5 \pm 0.5 \%$ for a solution-casted film to $7.8 \pm 0.8 \%$ for an electrospun mesh (Fig. 1e). Due to low thickness of the meshes $(h=50 \pm 2 \mu \mathrm{m})$, they appeared very delicate and ruptured easily in the thermomechanical cyclic tests, failing before reaching $\varepsilon_{\text {prog }}$ or during the zero-force shape-memory actuation experiments. Ruptures were not observed in SEM images of the programmed meshes due to high elasticity of the PLLA-PCL / ODLA blends, springing damaged fibers out of the mesh (Fig. 1b). Twisting them into a yarn contributed to the increase of the mechanical integrity of the non-woven materials by reducing the rate of

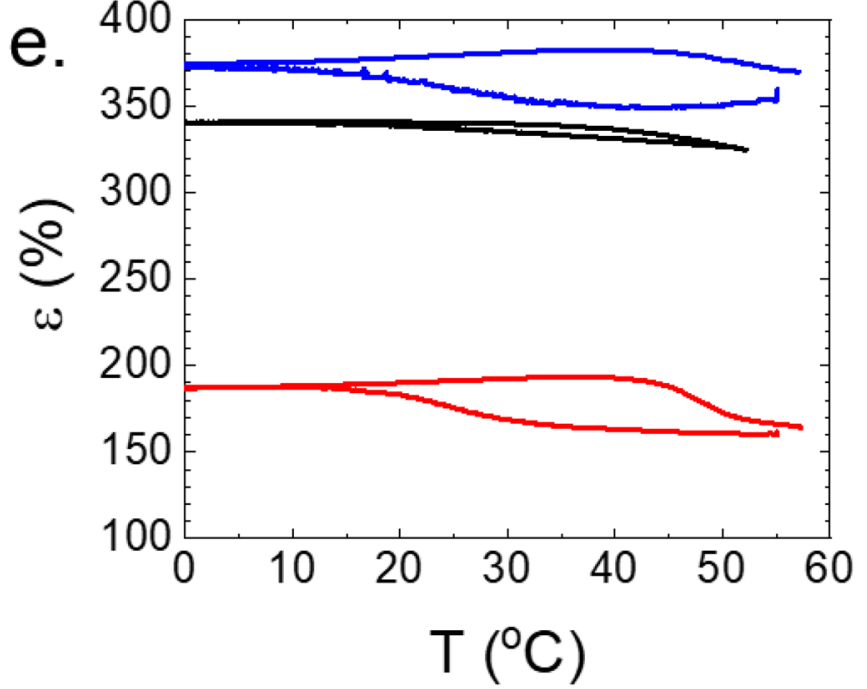

ing no preferential molecular orientation. e Shape-memory actuation diagrams of PLLA-PCL / ODLA. Black-solution-casted film, blue-electrospun mesh, red-twisted electrospun yarn. The hysteresis occurs i.a. due to the inequality of the melting and crystallisation temperature ranges

failures. This resulted in an improvement of $\varepsilon_{\text {rev }}^{\prime}$ to $15 \pm 1.8 \%$ (Fig. 1e). Additionally, this increase in $\varepsilon_{\mathrm{rev}}^{\prime}$ could be supported by the tendency of the twisted mesh to restore its flat shape upon heating, generating an additional axial force combining with the shape-memory actuation [18].

\section{Conclusions}

Physically cross-linked polymeric network can be formed by an established one-step electrospinning processing technique. Higher molecular orientation inherent to electrospun microfiber meshes, compared to the bulk material, has increased the shape-memory actuation performance in physically cross-linked PLLA-PCL / ODLA blends from 
$\varepsilon_{\text {rev }}^{\prime}=5.5 \pm 0.5 \%$ to $7.8 \pm 0.8 \%$. An improvement of structural integrity of electrospun meshes by twisting them into a yarn has resulted in further increase in $\varepsilon_{\text {rev }}^{\prime}$ to $15 \pm 1.8 \%$. The presented method allows for simultaneous orientating of polymer molecules and their cross-linking into a physical network, thereby outperforming covalently cross-linked fiber actuators, where the network is formed at the post-processing stage. The approach opens a broad perspective for the development of highly oriented and aligned fiber meshes.

Acknowledgements The authors thank Ms. Susanne Schwanz for her assistance with the WAXS measurements. Financial support was provided by the Helmholtz-Association through programme-oriented funding.

Funding Open Access funding enabled and organized by Projekt DEAL.

Data availability The datasets generated and analysed during the reported study are available from the corresponding author on a reasonable request.

\section{Declarations}

Conflict of interest A.L. and K.K. are co-inventors on patents and patent applications on shape-memory polymer actuators.

Open Access This article is licensed under a Creative Commons Attribution 4.0 International License, which permits use, sharing, adaptation, distribution and reproduction in any medium or format, as long as you give appropriate credit to the original author(s) and the source, provide a link to the Creative Commons licence, and indicate if changes were made. The images or other third party material in this article are included in the article's Creative Commons licence, unless indicated otherwise in a credit line to the material. If material is not included in the article's Creative Commons licence and your intended use is not permitted by statutory regulation or exceeds the permitted use, you will need to obtain permission directly from the copyright holder. To view a copy of this licence, visit http://creativecommons.org/licenses/by/4.0/.

\section{References}

1. A. Lendlein, O.E.C. Gould, Nat. Rev. Mater. 4(2), 116 (2019)

2. M. Behl, K. Kratz, J. Zotzmann, U. Nochel, A. Lendlein, Adv. Mater. 25(32), 4466 (2013)

3. K. Dušek, W. Prins, Adv. Polym. Sci. 6, 1 (1969)

4. M.K. Beyer, H. Clausen-Schaumann, Chem. Rev. 105, 2921 (2005)

5. S. Nikolov, R. Lebensohn, D. Raabe, J. Mech. Phys. Solids 54, 1350 (2006)

6. M. Farhan, D. Chaudhary, U. Nöchel, M. Behl, K. Kratz, A. Lendlein, Macromol. Mater. Eng. 306(2), 2000579 (2020)

7. T. Sauter, K. Kratz, M. Heuchel, A. Lendlein, Mater. Des. 202, 109546 (2021)

8. S. Pandini, S. Agnelli, A. Merlettini, F. Chiellini, C. Gualandi, K. Paderni, M.L. Focarete, M. Messori, M. Toselli, Macromol. Mater. Eng. 302(8), 1600519 (2017)

9. Q. Zhang, T. Rudolph, A.J. Benitez, O.E.C. Gould, M. Behl, K. Kratz, A. Lendlein, Smart Mater. Struct. 28(5), 055037 (2019)

10. F. Zhang, Z. Zhang, Y. Liu, H. Lu, J. Leng, Smart Mater. Struct. 22(8), 085020 (2013)

11. F. Zhang, Z. Zhang, Y. Liu, J. Leng, Fibers Polym. 15(3), 534 (2014)

12. H. Tsuji, Macromol. Biosci. 5, 569 (2005)

13. V. Izraylit, O.E.C. Gould, T. Rudolph, K. Kratz, A. Lendlein, Biomacromol 21(2), 338 (2020)

14. V. Crescenzi, G. Manzini, G. Calzolari, C. Borri, Eur. Polym. J. 8(3), 449 (1972)

15. S. Koombhongse, W.X. Liu, D.H. Reneker, J. Polym. Sci. Part B: Polym. Phys. 39(21), 2598 (2001)

16. K. Wasanasuk, K. Tashiro, M. Hanesaka, T. Ohhara, K. Kurihara, R. Kuroki, T. Tamada, T. Ozeki, T. Kanamoto, Macromolecules 44(16), 6441 (2011)

17. M.D. Edwards, G.R. Mitchell, S.D. Mohan, R.H. Olley, Eur. Polym. J. 46(6), 1175 (2010)

18. Q. Yang, J. Fan, G. Li, Appl. Phys. Lett. 109(18), 183701 (2016) 